\title{
DETECTION OF MOLECULAR MICROWAVE TRANSITIONS IN THE 3 MM WAVELENGTH RANGE IN COMET KOHOUTEK (1973f)
}

\author{
D. Buhl, W. F Huebner* and L. E. Snyder
}

Introduction

We have recently reported detection of hydrogen cyanide and the first quantitative observations of the velocities of neutral gas jets in the inner part of the coma while the comet was at small heliocentric distances (Huebner, et al., 1974). Now we report the detection of two line transitions from unidentified cometary molecules, provide further evidence of the variability of neutral gas jets, and give a summary of our search program for microwave transitions in molecules of cometary origin.

The observations presented here were made with a 3-mm line receiver mounted on the $11-\mathrm{m} \mathrm{NRAO}{ }^{* * *}$ radio dish at Kitt Feak. Observations were carried out before perihelion (15 to 20 December 1973) and after perihelion ( 3 to 7 January 1974)。During these periods the comet was between 0.3 and $0.5 \mathrm{AU}$ heliocentric distance. The antenna half-power beam width at $3 \mathrm{~mm}$ wavelength is $\theta_{\mathrm{B}} \approx 80 \mathrm{arc} \mathrm{s}$. The observations are based on data obtained from filter banks with a resolution of $250 \mathrm{kHz}$ and $100 \mathrm{kHz}$. Small local oscillator frequency offsets were made to check for system-generated signals. Searches at off-comet positions were carried out to obtain comparison spectra for noise determination. Comet velocity and position was obtained from ephemerides calculated independently by T. Clark (Goddard Space Flight Center) and Rh. Llust (Max-Planck-Institut flur Astrophysik,

*Work performed under the auspicles of the Energy Research and Development Administration

it The NRAO is operated by Associated Universities, Inc., under contract with the NSF 
Munich). The two sets of ephemerides agreed to within the pointing accuracy (10 arc s) of the telescope.

Table 1 summarizes the observational data. The columns, in order, list the UT date of observation, average comet position (RA and Dec) during each period of observation, molecular transition searched for, total integration time $(\delta t)$, single-sideband $r_{\mathrm{om}} \mathrm{s}$. system temperature $\left(\mathrm{T}_{\mathrm{S}}^{*}\right)$ obtained from all calibrations made during the integration interval, heliocentric distance $(r)$ of the comet, geocentric distance $(\Delta)$ of comet, geocentric radial velocity component $(\triangle)$ of comet, and the rest frequency $\left(\nu_{0}\right)$ of the theoretically strongest component of the molecular transition.

\section{Hydrogen Cyanide}

The detection of $\mathrm{HCN}$ has been reported earlier (Huebner, et al。, 1974). Here we present the spectral data of the $\mathrm{H}^{12} \mathrm{C}^{14} \mathrm{~N} J=1-0$ transition with more time resolution to show the variability of the gas jets and consistency of this phenomenon in other mother molecules. Figure 1(A) illustrates the average composite spectrum obtained December 15 and 16. In Figs. 1(B) and 1(C) the same transitions are presented as observed on January 3 and 6 . The bars above each spectrum indicate the frequencies of the three hyperfine quadrupole components $F=0-1,2-1$, and $1-1$, belonging to the same Doppler shifted velocity group measured with respect to the rest frame of the comet's nucleus. These laboratory frequencies as measured by DeLucia and Gordy (1969) are $88.63394 \mathrm{GHz}, 88.63185 \mathrm{GHz}$, and 


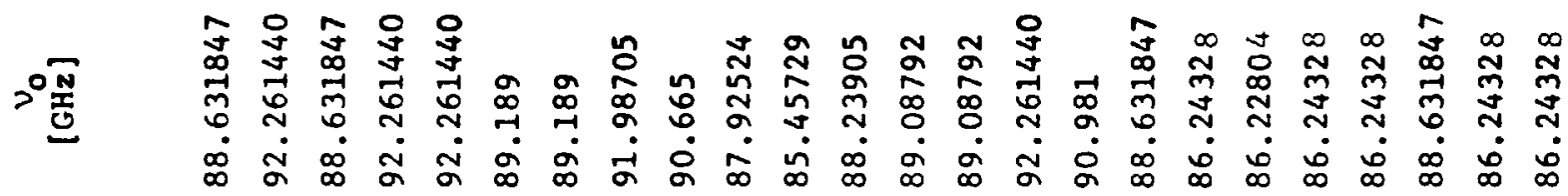

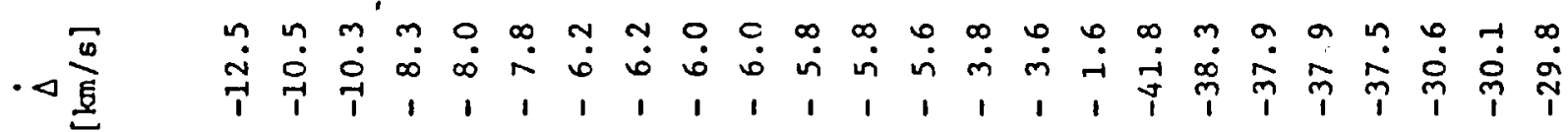

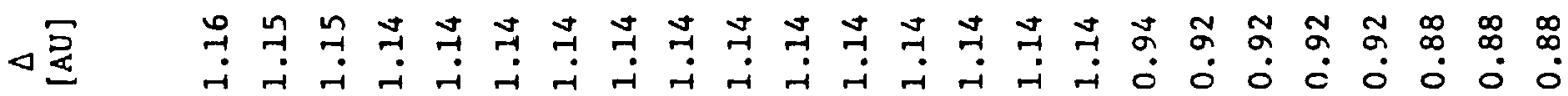

म完

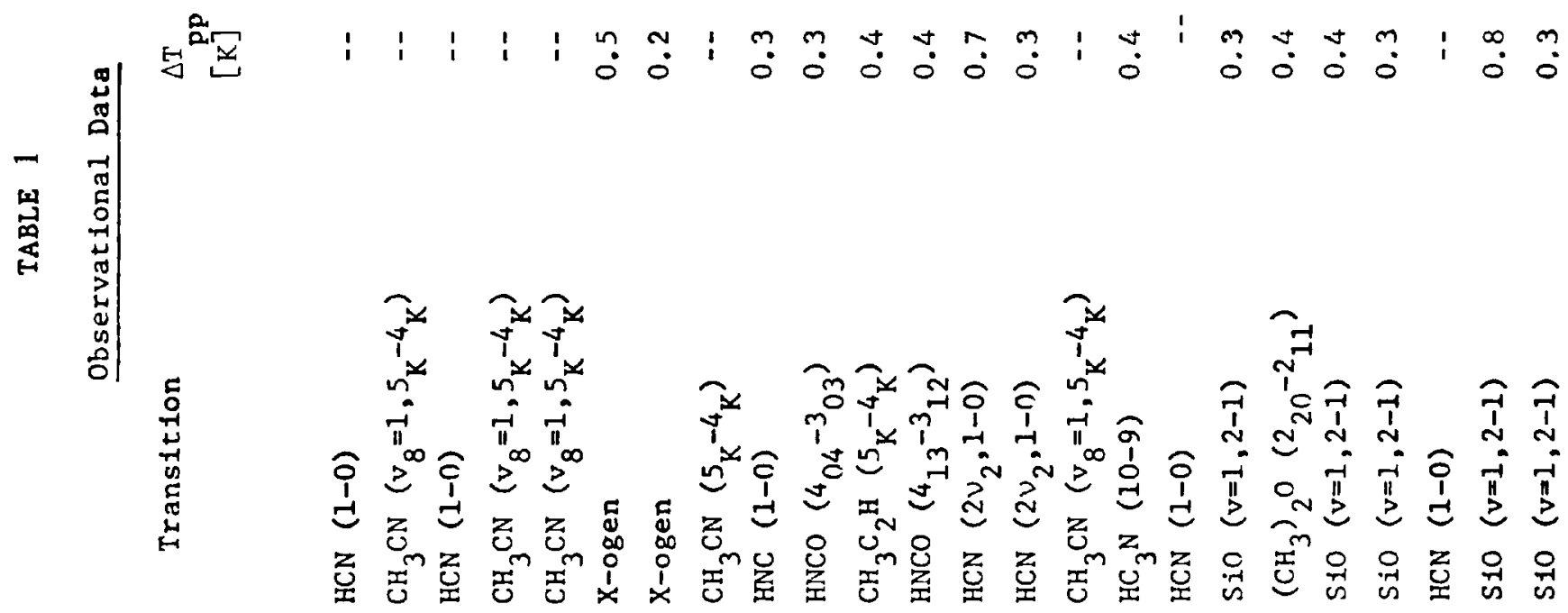

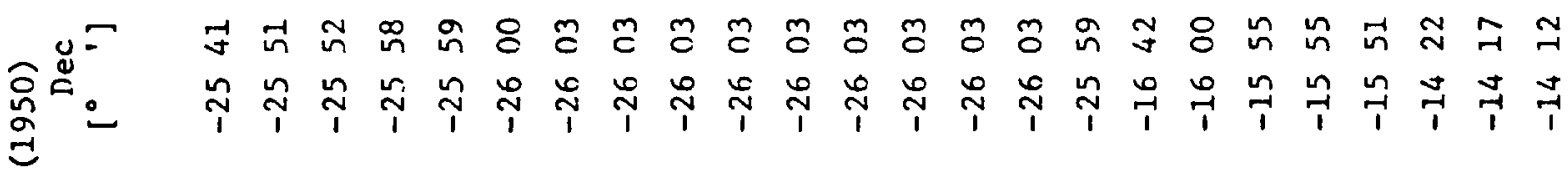

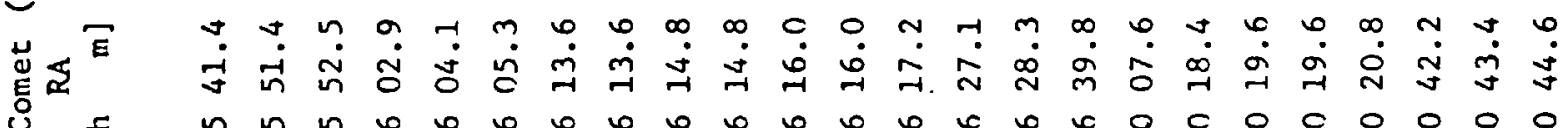

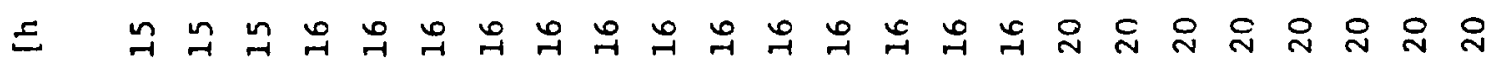

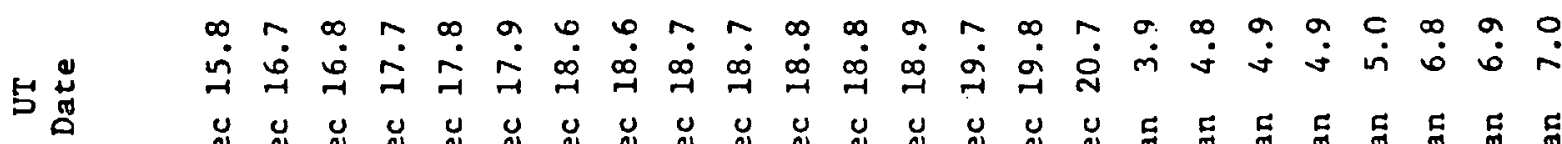



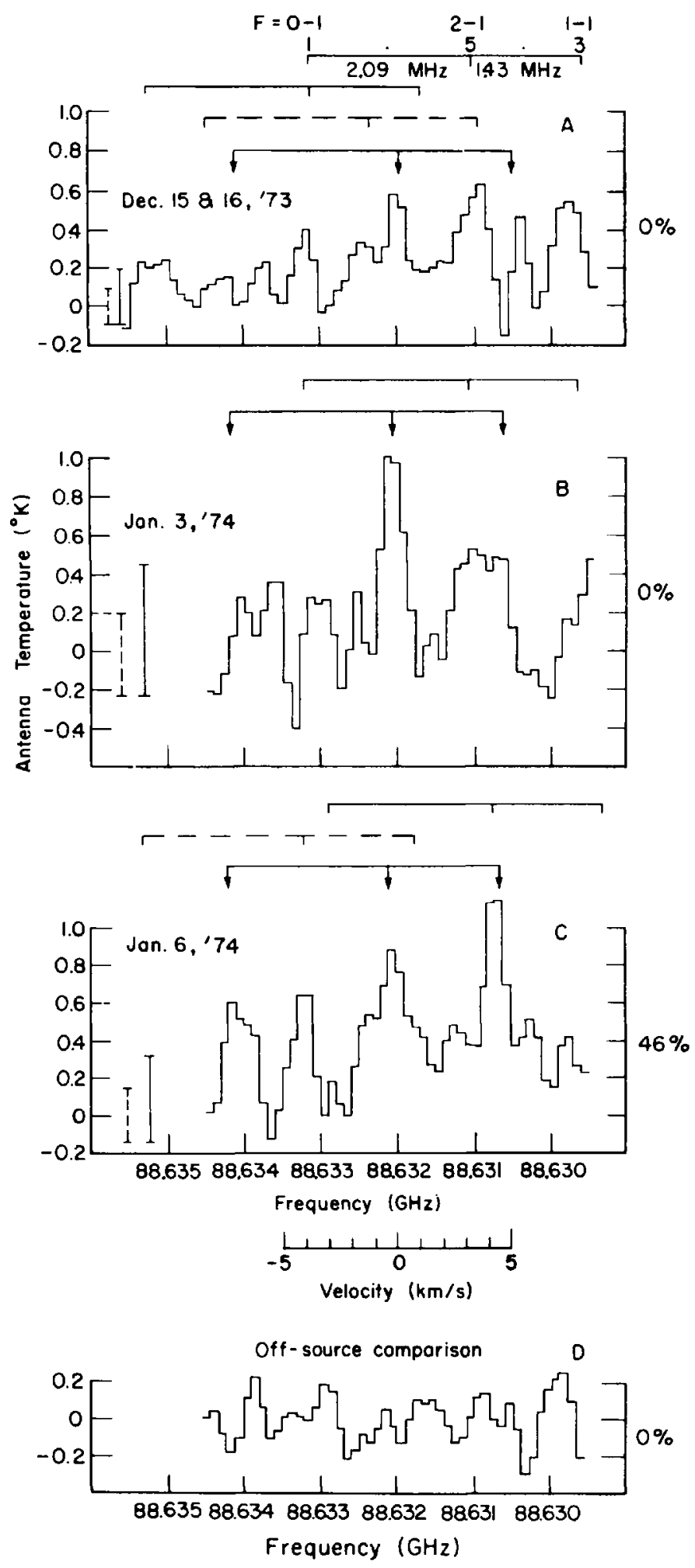

Figure 1: Emission spectrum of the HCN J = 1-0 transition observed in comet Kohoutek (1973 $\mathrm{f}$ ) before perihelion (A) and after perihelion (B) and (C). 
88. $63042 \mathrm{GHz}$ 。 Within each group the observed intensities follow closely the predicted theoretical values $(2 F+1)$ indicated above the top bar. The triplet with zero Doppler shift (within the width of a channel) with respect to the nucleus is indicated by arrows. It appears to be present in each spectrum, but fluctuates with time. The intensity of the other triplets also fluctuates with time, but in addition they change their number and frequency shift. The transitions with zero Doppler shift can be interpreted as a quiescent outstreaming of slowly released gas, however, their time variation suggests that they are also outbursts with velocities consistent with the other Doppler shifts, but close to the plane perpendicular to the earth-comet direction. There is no discernable decrease of intensity in the post-perihelion observations. Only the strongest Doppler shifted components of the spectrum are identified, possible weaker ones are indicated by dashed bars. Doppler shifts up to $\sim 1.3 \mathrm{MHz}\left(\sim 4 \mathrm{kms}^{-1}\right)$ can be measured. Figure $1(D)$ is a spectrum taken while tracking $\sim 7.5$ arc min off the comet nucleus and was used to determine the peak-to-peak noise which was found to be $\sim 0.3^{\circ} \mathrm{K}$. The peak-to-peak noise is indicated by two error bars in Figure 1 . The dotted error bar indicates the noise with the dome open, the solid error bar indicates the increase in noise due to dome attenuation. The percentage of time during which observations were made with the dome open is given to the right. 
Methyl Cyanide

Following the unexpected detection of ${ }^{12} \mathrm{CH}_{3}{ }^{12} \mathrm{C}^{14} \mathrm{~N}$ in its $\mathrm{v}_{8}=1$ excited state, transition $J_{K}=6_{3}-5_{3}$ by Ulich and Conklin (1974) on 1 and 5 December, 1973, we searched for the next lower rotational transition in the same vibrational state on 16, 17, and 19 December。 Although signals 3 to 4 times peak-to-peak noise were detected in the $100 \mathrm{kHz}$ filter bank the variability in intensity and frequency of the Doppler shifted lines resulted in identification problems: Doppler shifts similar to those measured in HCN could also be infered in the $\mathrm{CH}_{3} \mathrm{CN}$ spectrum, but since the spacing of its $\mathrm{K}$-component lines is bigger than the hyperfine splitting of the HCN spectrum one or the other of two $\mathrm{K}$-components was frequently shifted out of the range of the $100 \mathrm{kHz}$ filter bank. For this reason we present the analysis of the $\mathrm{CH}_{3} \mathrm{CN}$ spectrum in the $250 \mathrm{kHz}$ filter banks.

The average of the spectra obtained by Ulich and Conklin on 1 and 5 December with the $100 \mathrm{kHz}$ filter banks are presented in Fig. 2(A). The frequency scale has been reversed to facilitate comparison of their observation of the $J_{K}=6_{0}-5_{0}$ and $6_{3}-5_{3}$ transition with our $J_{K}=5_{0}-4_{0}$ and $5_{3}-4_{3}$ observations.

Figure 2(B-D) presents our observed spectrum of ${ }^{12} \mathrm{CH}_{3}{ }^{12} \mathrm{C}^{14} \mathrm{~N}$ in the $\mathrm{v}_{8}=1$ excited state. The transitions correspond to $\mathrm{J}_{\mathrm{K}}=5_{2}-{ }_{2}$, $5_{0}-4_{0}, 5_{3}-4_{3}$, and $5_{1}-4_{1}$. The corresponding frequencies as measured by Bauer and Maes (1969) are $92.26399 \mathrm{GHz}, 92.26144 \mathrm{GHz}$, 

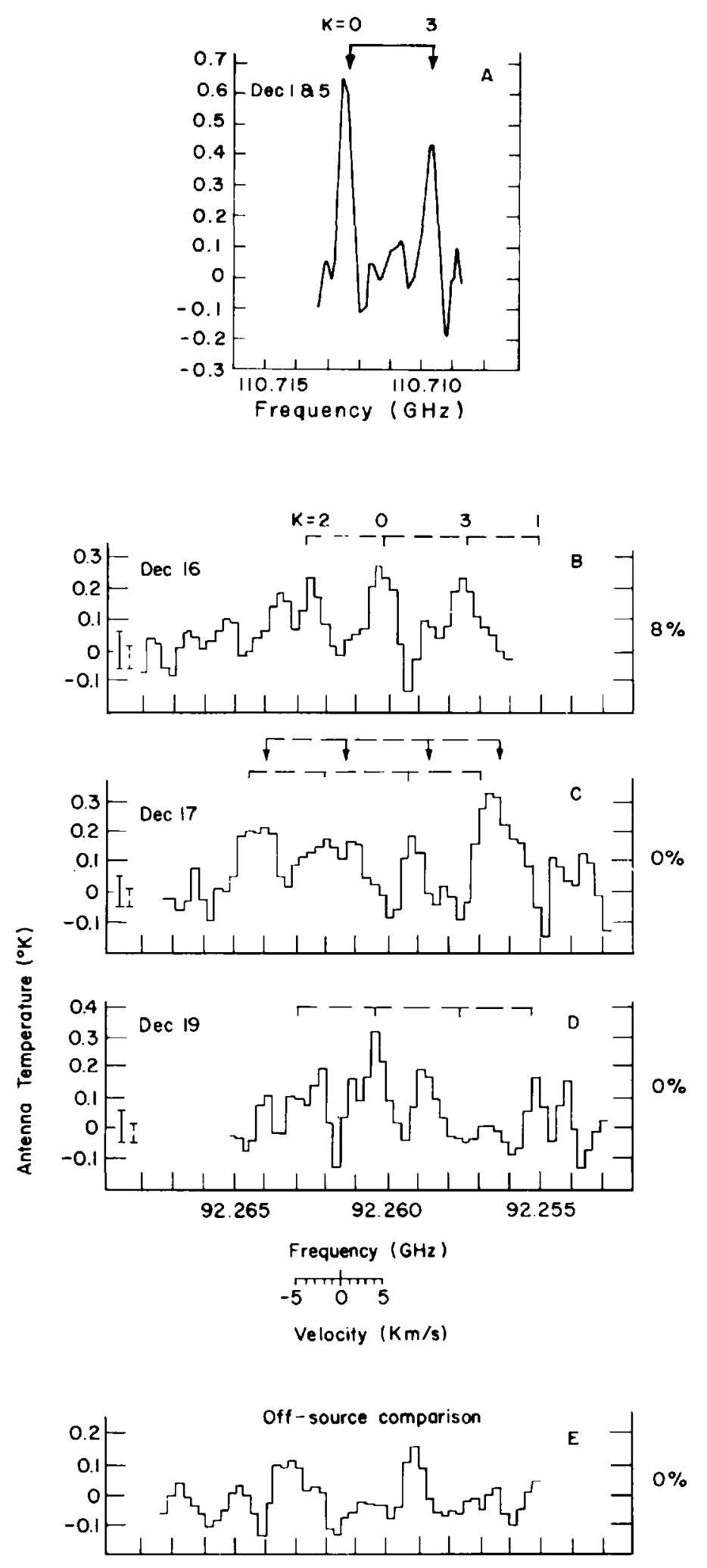

Figure 2: Emission spectrum of the $\mathrm{CH}_{3} \mathrm{CN} \mathrm{v}$. 1 state observed in the comet in the $\mathrm{J}_{\mathrm{K}}=6_{0}-5_{0}$ and $6_{3}-5_{3}$ transitions (A) and the $J_{\mathrm{K}}=5_{\mathrm{K}}-4_{\mathrm{K}}, \mathrm{K}=0,1,2$, and 3 transition (B), (C), and (D). 
$92.25841 \mathrm{GHz}$ and $92.25629 \mathrm{GHz}$. Doppler shifts up to about $\pm 1.0 \mathrm{MHz}$ are measured. The zero point of the velocity scale below Fig. 2(D) indicates the expected position of the $\mathrm{K}=0$ component under quiescent conditions. Bars above each spectrum connect $\mathrm{K}$-components exhibiting the same Doppler shift; the bar with arrows pointing downward indicates transitions of the "quiescent" state with nearly zero Doppler shift. It should be noted that the "quiescent" state is not always present, which strengthens the interpretation that it is due to jets in a plane perpendicular to the line of sight rather than a uniform outgassing. The spectra as presented in Fig. 2(A-D) get progressively weaker. Only our 16 and possibly 17 December spectra are strong enough to serve as confirmation of the detection of $\mathrm{CH}_{3} \mathrm{CN}$ by $\mathrm{UIich}$ and Conklin. The signal to noise ratio is insufficient for a direct and independent identification of the molecule.

On 18 December we made a search for ${ }^{12} \mathrm{CH}_{3}{ }^{12} \mathrm{C}^{14} \mathrm{~N}$ vibrational ground state $\mathrm{J}_{\mathrm{K}}=5_{\mathrm{K}}-4_{\mathrm{K}}$ transitions simultaneously with a search for X-ogen (Buh1 and Snyder, 1970) in the other side-band of the receiver. There was a possible detection of weak components $\left(|\mathrm{K}|=3,2,0\right.$, and 1 ) at about $\mathrm{T}_{\mathrm{A}}{ }^{*}=0.4 \mathrm{~K}$. The corresponding rest frequencies measured by Bauer and Maes (1969) are $91.97137 \mathrm{GHz}$, $91.98000 \mathrm{GHz}, 91.98528 \mathrm{GHz}$ and $91.98705 \mathrm{GHz}$.

Two unidentified lines

On 3 January Snyder and Buhl (1974) discovered a peculiar masering transition with several frequency components as a point source 
in Orion. A search for these lines was made in the comet on $4,5,6$, and 7 of January with several shifts of the local oscillator frequency. As a result of this search two lines were acquired one in the upper side-band of the $250 \mathrm{kHz}$ filter bank receiver at $89.0105 \mathrm{GHz}$ and one in the lower side-band at $86.2471 \mathrm{GHz}$. The summary of these observations is presented in Fig. 3(A and $\mathrm{B}$ )。 The interstellar lines were later identified as Doppler shifted components of SiO with rest frequency $86.24328 \mathrm{GHz}$ corresponding to the transition $\mathrm{v}=1$, $\mathrm{J}=2-1$ (Snyder and Buh1, 1974) and cannot be brought into agreement with the lines observed in the comet. There are no known transitions in the neighborhood of $89.0105 \mathrm{GHz}$. The frequency of the other line $(86.2471 \mathrm{GHz})$ is close to that of ethanol ( $86.2474 \mathrm{GHz})$ and acetone $(86.2479 \mathrm{GHz})$, but probably cannot be identified with either one of these molecules for the following reasons: (A) The line is too broad, indicating an approximately isotropic expansion velocity of $\sim 3 \mathrm{kms}^{-1}$. This requires the additional assumption that an exothermic process took place. (B) The line does not exhibit the resolvable Doppler shifted components and thus is not consistent with the $\mathrm{HCN}$ and $\mathrm{CH}_{3} \mathrm{CN}$ observations made at about the same heliocentric distance. (C) If the molecule were acetone one would also expect to find a line at $86.2447 \mathrm{GHz}$ which is not observed. The source of the two unidentified lines is probably a radical which during the process of decay of its mother molecule received an excess of kinetic energy as, e.g., can occur during photodissociation. 


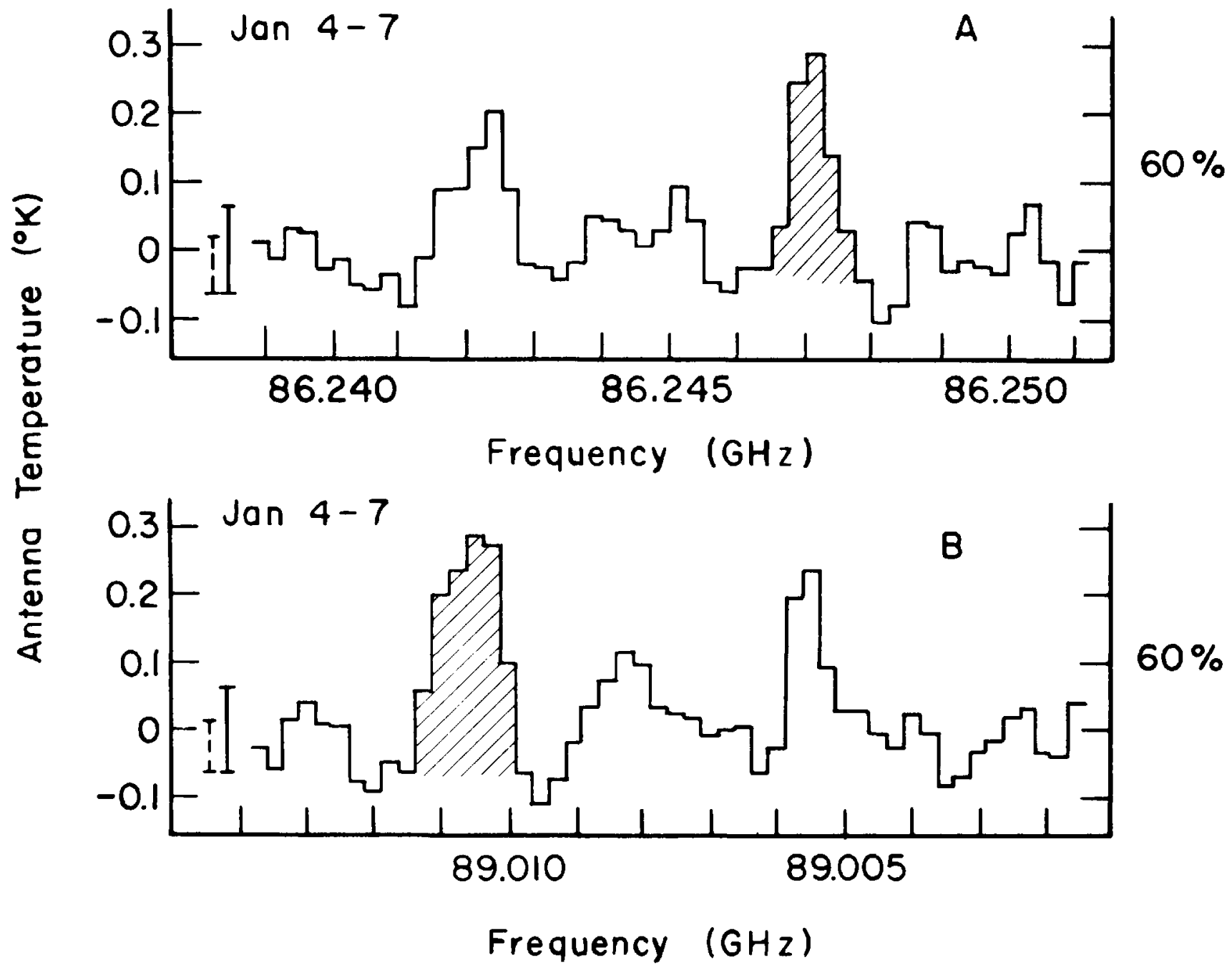

Fig. 3. Two unidentified line transitions found during the Si 0 search in the lower side band (A) at $86.2471 \mathrm{GHz}$ and In the upper side band (B) at $89.0105 \mathrm{GHz}$. Since lower and upper side band of the recelver are super imposed in the display but with frequencies increasing in opposite directions the line transitions show up in both spectra (A) and (B) but the side band to which they belong can be assigned uniquely through shifts in the local oscillator frequency. 


\section{Production Rates}

Figure 4 presents the earth's orbit and the projection of the comet's orbit on the ecliptic and indicates the times and heliocentric distances when the above-mentioned observations were carried out. Ulich and Conklin observed between 1 and 5 December when the comet was at heliocentric distances between 0.87 and $0.79 \mathrm{AU}$. Their spectra indicate a quiescent production of methyl cyanide. Our observations of methyl cyanide were made when the comet was between 0.46 and $0.37 \mathrm{AU}$ heliocentric distance before perihelion. By that time the production was very weak and getting weaker. Discrete jets with speeds of several $\mathrm{km} / \mathrm{sec}$ with respect to the nucleus are measured from Doppler shifts. These indicate an inhomogeneous structure of the nucleus (Huebner, 1974, 1975). Observation of the spectrum in the vibrationally excited state $\sim 640{ }^{\circ} \mathrm{K}$ above the ground state, the lack of a Boltzmann distribution, and the action of jets make estimates for the abundance very difficult. In the absence of detailed knowledge about the excitation mechanism and the cross sectional area of the jets we apply a quiescent state fluid dynamic model (Huebner and Snyder, 1970) to our ground state observation.

The fluid model is valid as long as:

$$
\mathrm{A}<\mathrm{n} \text { v }
$$

where $A=$ Einstein emission coeff。 for microwave transition in $\sec ^{-1}$, $\mathrm{n}=$ number density of molecules $/ \mathrm{cm}^{3}, \mathrm{v}=$ escape velocity $\approx$ thermal velocity $\approx 3 \times 10^{4} \mathrm{~cm} / \mathrm{sec}$ and $\sigma=$ collision cross section $\approx 10^{-15} \mathrm{~cm}^{2}$. 
Kohoutek (1973f)

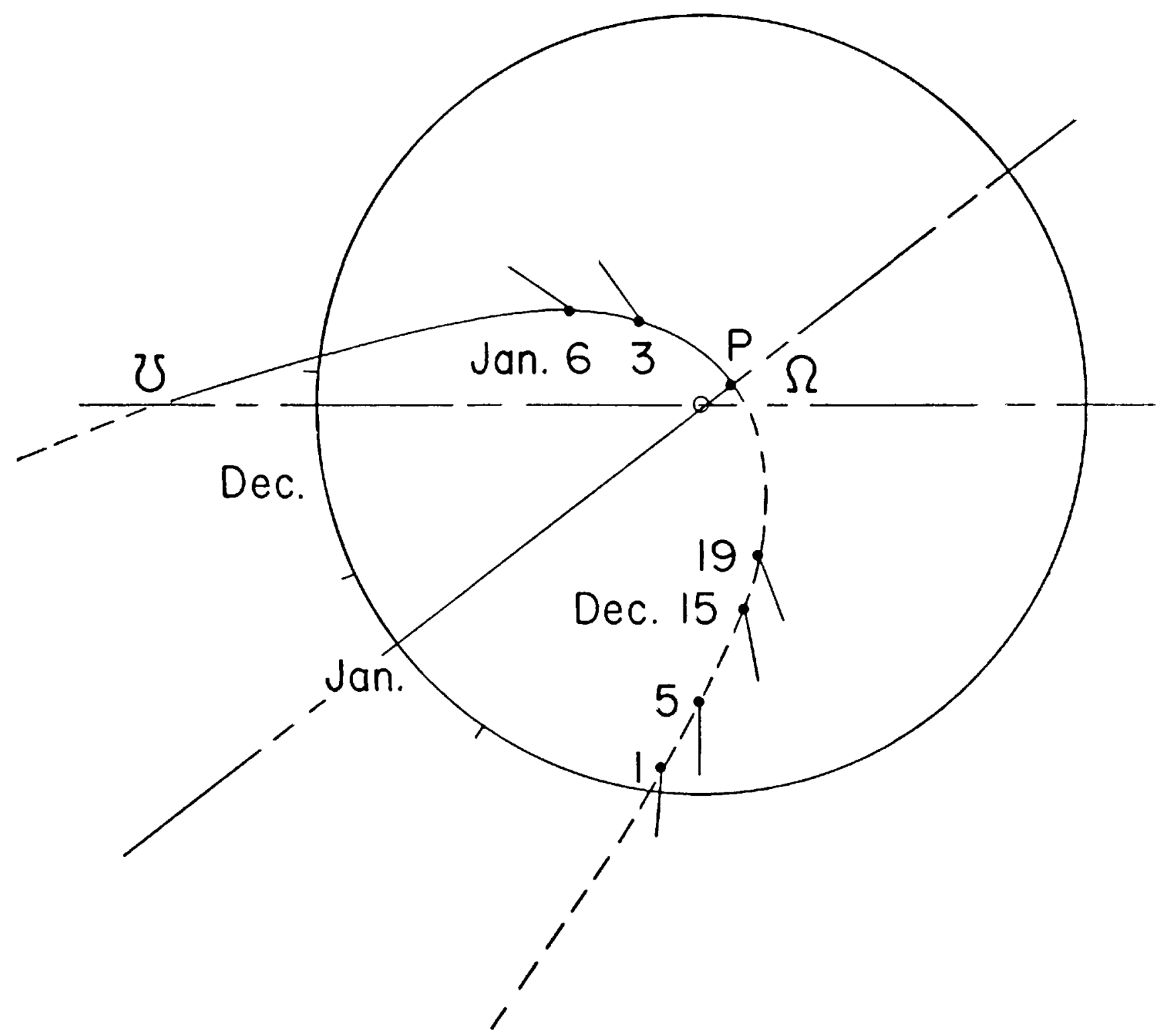

Fig.4. Projection of the orbit of the comet onto the ecliptic. Earth positions for December 1, January 1, and February 1 are indicated on the circle. Comet positions corresponding to the dates of observations by Ulich and Conklin and by us are shown on the parabola. 
For our fluid dynamic model

$$
\mathrm{n}=\frac{\mathrm{Z}}{\mathrm{v}}\left(\frac{\mathrm{R}}{\mathrm{r}}\right)^{2}
$$

Where $\mathrm{Z}=$ gas production rate of comet $\approx\left(10^{18 / \mathrm{r}_{\mathrm{h}}}{ }^{2}\right)$ molecules sterad ${ }^{-1}$ $\mathrm{cm}^{-2} \mathrm{sec}^{-1}$ for $r_{h} \leq 1 \mathrm{AU}$ where $r_{h}=$ heliocentric distance in $\mathrm{AU}$; $\mathrm{R}=$ radius of nucleus $\approx 3$ to $5 \times 10^{5} \mathrm{~cm}$ for Kohoutek, and $\mathrm{r}=$ radial distance of molecules in the coma as measured from center of nucleus.

$$
\begin{aligned}
\therefore A & <\frac{Z \sigma}{r_{h}^{2}}\left(\frac{R}{r}\right)^{2} \approx\left(1018 / r_{h}^{2}\right) \times 10^{-16}\left(10^{5.5} / r\right)^{2} \\
A & <10^{13} /\left(\mathrm{rr}_{h}\right)^{2}
\end{aligned}
$$

In our calculations $\mathrm{r}_{\mathrm{h}} \approx 0.3$ to $0.5 \mathrm{AU}$. We assumed two cutoff values for $\mathrm{r}: \mathrm{r}_{\mathrm{o}}=10^{4} \mathrm{~km}=10^{9} \mathrm{~cm}$ and $\mathrm{r}_{\mathrm{o}}{ }^{*}=10^{5} \mathrm{~km}=10^{10} \mathrm{~cm}$.

$$
\begin{aligned}
\therefore A & <10^{-3} \mathrm{sec}^{-1} \text { for } r_{0}=10^{4} \mathrm{~km} \\
A & <10^{-5} \mathrm{sec}^{-1} \text { for } r_{0}=10^{5} \mathrm{~km}
\end{aligned}
$$

The fluid model breaks down when the collision mean free path becomes larger than the distance traveled, i.e., it breaks down at $r \approx 1 /(n \sigma)$, or $r \approx \mathrm{ZR}^{2} \sigma / \mathrm{v} \approx 10^{10} \mathrm{~cm}$. Hence, within the cutoff radius which we consider the fluid model is valid.

The optical depth, frequency-averaged over the full width $(\Delta \nu)$ of a line at one-half the maximum line intensity, for a symmetric top 
molecule is

$$
(T)=\frac{16 \pi^{2} \ll \mathrm{N}>\mathrm{f}_{v} h^{3 / 2} \mu^{2} \nu^{2}(A B C)^{1 / 2}}{3 c \Delta \nu(k T)^{5 / 2}} \cdot \frac{(J+1)^{2}-k^{2}}{J+1} e^{-W / T} \text {, }
$$

for $(\mathrm{J}+1)_{\mathrm{K}} \rightarrow \mathrm{J}_{\mathrm{k}}$ 。

The effects of beam dilution, $\beta$, have been included, i。e,

$$
<T>\equiv \beta \bar{\tau},
$$

and

$$
<\mathrm{N}>\equiv \beta \mathrm{N}
$$

Here $\mathrm{N}$ is the column density of the total number of $\mathrm{CH}_{3} \mathrm{CN}$ molecules, $\mu$ is the dipole moment $\left(3.92 \times 10^{-18}\right.$ esu. $\mathrm{cm}$ for $\left.\mathrm{CH}_{3} \mathrm{CN}\right), \nu$ is the transition frequency, and A, B, and $\mathrm{C}$ are the rotational constants $\left(\mathrm{A}=\mathrm{B}=9.199 \mathrm{GHz}, \mathrm{C}=158.0 \mathrm{GHz}\right.$ and $\left.\mathrm{CH}_{3} \mathrm{CN}\right)$. The rotational energy of the upper level $\mathrm{W}=13.2^{\circ} \mathrm{K}$ for $\mathrm{J}_{\mathrm{K}}=5_{0}-{ }^{4} 0$ in $\mathrm{CH}_{3} \mathrm{CN}$ enters the assumed Boltzmann distribution for the vibrational ground state for which the partition function is $\mathrm{f}_{\mathrm{v}} \approx 1$.

If the comet coma is optically thin and its $3 \mathrm{~mm}$ continuum emission is negligible then the antenna temperature, $\mathrm{T}_{\mathrm{A}}^{*}$, as measured by chopper whee 1 calibration is related to the optical depth, Eq. (5), by

$$
\left.\mathrm{T}_{\mathrm{A}}^{*}=\mathrm{T}<\mathrm{T}\right\rangle
$$

If emission is not enhanced (which is apparently not true for the $v_{8}=1$ state) by collisional or radiative excitation, then the 
temperature is determined by the vaporization equilibrium, $\mathrm{T} \approx 150{ }^{\circ} \mathrm{K}$. An upper limit for the $\mathrm{K}=0$ component is $\mathrm{T}_{\mathrm{A}}^{*} \approx 0.4{ }^{\circ} \mathrm{K}$. This gives for the optical depth including beam dilution $\langle T\rangle \approx 0.0027$. Although smoothing gives the lines a much broader appearance, the total halfintensity line width $\Delta \nu \approx 200 \mathrm{kHz}$. Substitution into Eq. (1) gives for the column density including beam dilution $\langle\mathbb{N}\rangle \approx 1.6 \times 10^{13} \mathrm{~cm}^{-2}$ as an approximate upper limit. The range of the methyl cyanide molecules before they are destroyed by photodissociation or photoionization is not known. Two typical values will be assumed for the cutoff range, $r_{0}=10^{4}$ and $10^{5} \mathrm{~km}$ 。 The column density uniformly distributed over the antenna beam is

$$
\left\langle N>=\frac{4 Q^{\prime}}{\pi v \Delta^{2} \theta_{B}^{2}}\left[s \cos ^{-1} \frac{s}{r_{0}}-\left(r_{o}^{2}-s^{2}\right)^{1 / 2}+r_{0}\right]\right.
$$

with

$$
s=\min \left[r_{0}, \Delta^{\circ} \theta_{B} / 2\right]
$$

Here $Q^{\prime}$ is the total production rate of the molecule under consideration, $\mathrm{v} \approx 0.3 \mathrm{kms}^{-1}$ is the average (thermal) expansion velocity of the escaping gas, $\theta_{B}$ is the half-power antenna beam width, and $\Delta$ is the geocentric distance of the comet. Using $\Delta=1.14 \mathrm{AU}$ an upper 1 imit for the production rate of $\mathrm{CH}_{3} \mathrm{CN}$ at $r=0.40 \mathrm{AU}$ heliocentric distance is $Q^{\prime}=1.7 \mathrm{x}$ $10^{28} \mathrm{~s}^{-1}$ for $r_{0}=10^{4} \mathrm{~km}$ and $\mathrm{Q}^{\prime}=3.6 \times 10^{27} \mathrm{~s}^{-1}$ for $\mathrm{r}_{\mathrm{o}}=10^{5} \mathrm{~km}$ 。 The production rate of $\mathrm{HCN}$ based on observations of the $J=1-0$ vibrational ground state transitions was reported earlier by Huebner, 
et a1., (1974) to be $Q^{\prime}=1.2 \times 10^{28} \mathrm{~s}^{-1}$ for the cutoff range $r_{0}=10^{4} \mathrm{~km}$ and $Q^{\prime}=3 \times 10^{27} \mathrm{~s}^{-1}$ for $r_{o}=10^{5} \mathrm{~km}_{0}$ No spectrum could be detected in the vibrationally excited $2 v$ state. Upper limits for production rates of other molecules listed in Table 1 can be obtained, taking into account the appropriate molecular symmetry properties in the calculation of the optical depth (see, e.g。, Townes and Schawlow, 1955). The results are summarized in Table 2.

Production rates are related to abundances of the constituents in the frozen state, but latent heats of vaporization and likely inhomogenities in the structure of the nucleus must be considered in such an analysis. It should be noted that, with the sole exception of water, only molecules with strong transition probabilities in the radio range have been detected. Other molecules may be more abundant, yet their detection is more difficult because of weak transition probabilities or high latent heats of vaporization. The difficulties encountered with the detection of water by Jackson, et al., (1975) is a typical example.

\section{Acknowledgements}

We wish to thank Dr. W. Howard for granting observing time on the $11 \mathrm{~m}$ telescope and NRAO personnel at Kitt Peak for their support during the observations. We were assisted with telescope operations by D. Cardarella, D。 Myers, P。Rhodes and C. Sparks。 It is a pleasure to acknowledge the help we received from Drs. T. Clark, 


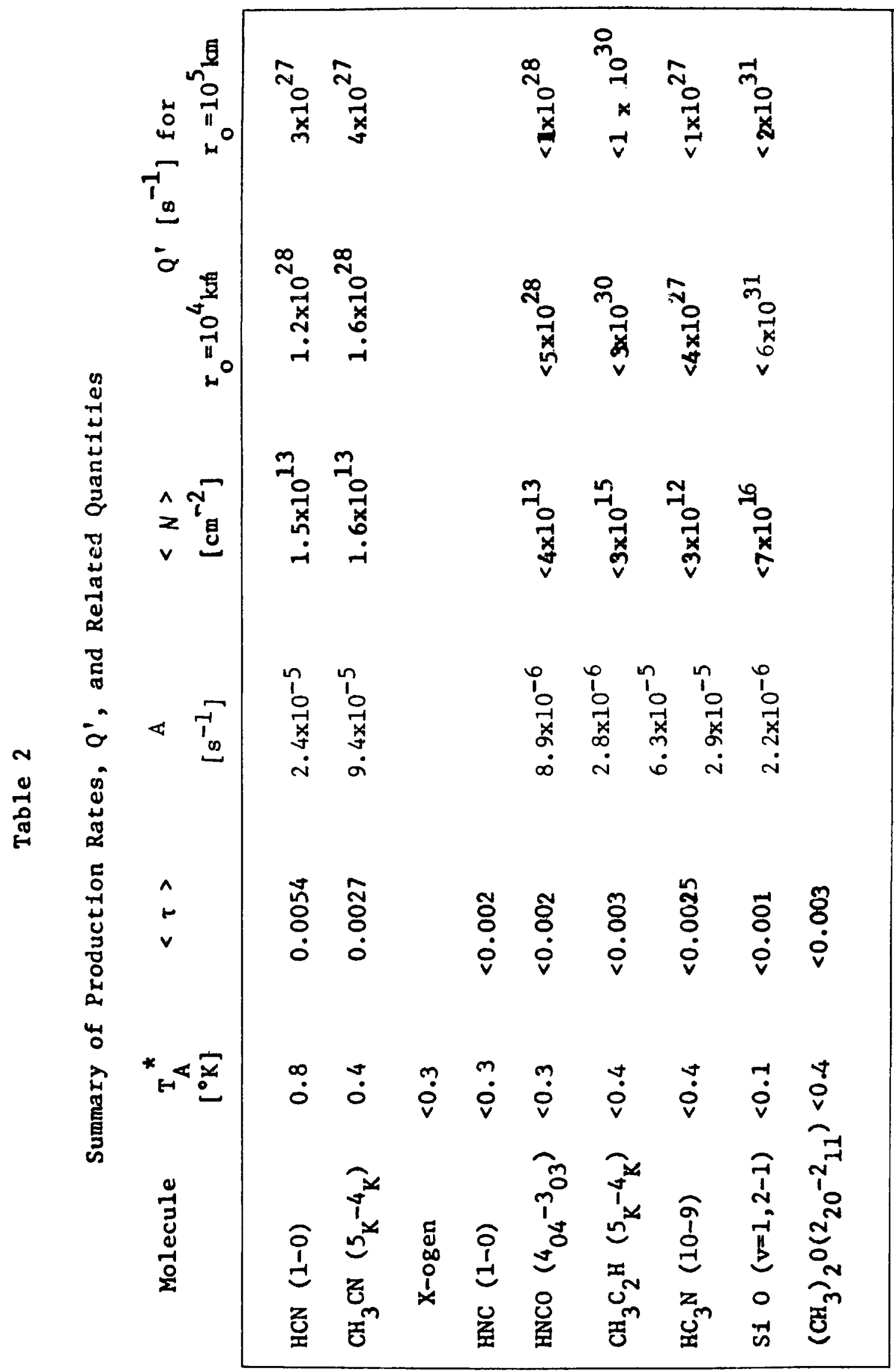


Rh. Llust, and B. G. Marsden on the ephemerides, and M.L. Stein for invaluable help in the reduction of the data on the MANIAC II computer at the LASL。 L。E.S. received partial financial support during this work from NSF grant GP-34200 to the University of Virginia. 


\section{REFERENCES}

Bauer, A., and Maes, S. (1969) J. Phys. (Paris) 30, 169.

Buh1, D., and Snyder, L. E. (1970) Nature 128, 267.

Delucia, F., and Gordy, W. (1969) Phys。Rev。 187, 58.

Huebner, W.F。, and Snyder, L。E。(1970) Astron。 J. 75, 759.

Huebner, W.F。, Snyder, L。E., and Buh1, D. (1974) Icarus, 23, 580 。

Huebner, W.F。(1974) Comet Kohoutek Workshop, NASA/MSFC, NASA

Special Publication, in press.

Huebner, W.F. (1975) I.A.U。Colloquium No. 25, to be published.

Jackson, W.M., Clark, T., and Donn, B. (1975) I.A.U. Colloquium No. 25, to be published.

Snyder, L。E。 and Buh1, D。(1974) Ap。J. (Letters), 189, L31。

Townes, C.H., and Schawlow, A.L。 (1955) Microwave Spectroscopy,

McGraw-Hill Book Company, New York, Toronto, London.

Ulich, B.L., and Conklin, E.K. (1974) Nature 248, 121。 